\title{
Continuous anti-angiogenic therapy after tumor progression in patients with recurrent high-grade epithelial ovarian cancer: phase I trial experience
}

\author{
Ming-Mo Hou ${ }^{1,3, *}$, Zhijie Wang ${ }^{4, *}$, Filip Janku' ${ }^{1}$, Sarina Piha-Paul, Aung Naing ${ }^{1}$, \\ David Hong $^{1}$, Shannon Westin ${ }^{2}$, Robert L. Coleman², Anil K. Sood ${ }^{2}$, Apostolia M. \\ Tsimberidou $^{1}$, Vivek Subbiah ${ }^{1}$, Jennifer Wheler ${ }^{1}$, Ralph Zinner ${ }^{1}$, Karen Lu ${ }^{2}$, Funda \\ Meric-Bernstam ${ }^{1}$, Siqing Fu ${ }^{1}$ \\ ${ }^{1}$ Departments of Investigational Cancer Therapeutics, The University of Texas MD Anderson Cancer Center, Houston, Texas, \\ USA \\ ${ }^{2}$ Departments of Gynecologic Oncology and Reproductive Medicine, The University of Texas MD Anderson Cancer Center, \\ Houston, Texas, USA \\ ${ }^{3}$ Division of Hematology-Oncology, Chang Gung Memorial Hospital and Chang Gung University, Taoyuan, Taiwan \\ ${ }^{4}$ Department of Thoracic Medical Oncology, Key Laboratory of Carcinogenesis and Translational Research (Ministry of \\ Education), Peking University Cancer Hospital \& Beijing Institute for Cancer Research, Beijing, China \\ "These authors have contributed equally to this work \\ Correspondence to: Siqing Fu, e-mail: siqingfu@mdanderson.org \\ Keywords: epithelial ovarian cancer, anti-angiogenesis, tumor progression, progression-free survival, overall survival \\ Received: March 01, $2016 \quad$ Accepted: April 10, $2016 \quad$ Published: April 27, 2016
}

\section{ABSTRACT}

High-grade epithelial ovarian cancer (HG-EOC) is the most lethal gynecologic malignancy worldwide Once patients develop chemoresistance, effective novel strategies are required to improve prognosis We analyzed characteristics and outcomes of 242 consecutive patients with HG-EOC participating in 94 phase I clinical trials at The University of Texas MD Anderson Cancer Center. Baseline lactate dehydrogenase levels, albumin levels, and number of metastatic sites were independent predictors of overall survival (OS). Receiving more than 1 phase I protocol was associated with improved OS $(p<0.001)$. Regimens including a chemotherapeutic agent plus bevacizumab or Aurora A kinase inhibitor led to a median progression-free survival (PFS) duration of more than 6 months. Although patients receiving bevacizumabbased regimens in the phase I clinical trials had significantly longer PFS than those receiving other anti-angiogenic therapies $(p=0.017)$, patients treated with vascular endothelial growth factor receptor-tyrosine kinase inhibitors (VEGFR-TKIs) had significantly longer OS (12.2 months) than those not treated with VEGFR-TKIs (8.6 months, $\mathrm{p}=\mathbf{0 . 0 1 5}$ ).

In conclusion, anti-angiogenic therapy is one of the most important strategies for the treatment of HG-EOC, even in those who have already experienced tumor progression. Therefore, eligible patients with HG-EOC should be encouraged to participate in novel phase I studies of anti-angiogenic therapies, even after disease progression.

\section{INTRODUCTION}

Epithelial ovarian cancer (EOC) is the most lethal gynecologic malignancy worldwide and the fourth most common cause of cancer-related death in women [1]. As a major histologic type, high-grade EOC (HG-EOC) constitutes more than $80 \%$ of ovarian cancer and most frequently presents as advanced-stage disease with poor survival prognosis $[2,3]$. Cytoreductive surgery after neoadjuvant therapy or followed by platinum-based chemotherapy is a popular and effective management strategy $[4,5]$. However, despite satisfactory clinical 
outcomes after initial therapy, most patients suffer relapse $[6,7]$. Subsequent chemotherapy is only modestly effective, even for platinum-sensitive patients. Once patients develop chemoresistance, effective novel strategies are required to improve prognosis [6].

Although many new cytotoxic agents and therapeutic schedules are being developed, a therapeutic bottleneck appears to have been reached. The emergence of targeted therapeutic agents, based in part by an expanding understanding of the biological systems governing oncogenesis, brought promise to these patients [8]. The most extensively investigated targeted regimens have been anti-angiogenic agents, including the vascular endothelial growth factor (VEGF) antibody bevacizumab [9-12] and VEGF receptor-tyrosine kinase inhibitors (VEGFR-TKIs) [13-16]. Though a total of 8 phase III trials with these agents have been conducted in the frontline, maintenance and recurrent disease setting with uniform improvement in progression-free survival [6], bevacizumab is FDA-approved in combination with chemotherapy only for patients with platinum-resistant recurrent ovarian cancer [6]. Poly (adenosine diphosphate [ADP]-ribose) polymerase inhibitors have also been evaluated as part of single or combined therapy, and these demonstrated superior efficacy in EOC patients with germline BRCA1/2 (gBRCA-mt) mutations [17-19]. The FDA granted accelerated approval to olaparib, the PARP inhibitor, for the treatment of recurrent $g B R C A-m t$ EOC patients who have been treated with 3 or more prior lines of therapy. Other promising targeted agents developed for the treatment of EOC include agents targeting PI3K-AKTmTOR [20, 21], MAPK [22], Src [23, 24], Wee1 [25], and Aurora A kinase signaling pathways [26, 27].

Beyond investigations of safety and tolerance of newly developed targeted agents, phase I clinical trials provide the first step in the delivery of future potential therapeutic regimens. However, with many phase I clinical trials currently in progress and a growing number of patients with refractory disease, it is difficult to determine which treatments are most likely to lead to good outcomes and which patients would most benefit from enrolling in the trials. We here in report the clinical characteristics and outcomes of a large cohort of consecutive patients with advanced HG-EOC participating in phase I clinical trials at The University of Texas MD Anderson Cancer Center (MD Anderson), to identify the most promising potential novel regimens for the treatment of patients with HG-EOC.

\section{RESULTS}

\section{Patient characteristics}

In the cohort of 242 patients enrolled in phase I clinical trials, most patients were white $(80 \%)$, had good ECOG performance status of 1 or better $(96 \%)$, were diagnosed with serous carcinoma (79\%), and had stage III-IV disease $(90 \%)$ at their initial diagnosis (Table 1).
In patients who had molecular profiling performed, the most frequent gene aberration was a TP53 hotspot mutation (49 out of the 79 patients tested; $62 \%$ ). Other molecular aberrations included BRCA1/2 (8/18, 44\%), PIK3CA (17/190, 9\%), KRAS (15/168, 9\%), c-KIT (7/102, 7\%), KDR (3/54, 6\%), B-RAF $(3 / 163,2 \%)$, NRAS $(2 / 111,2 \%)$, AKT-1 (1/93, 1\%), EGFR (1/149, $1 \%$ ), and MET amplification (7/119, 6\%). No ALK rearrangement (84 patients tested), GNAS mutations (66 patients tested), or GNAQ mutations (54 patients tested) were detected. Fluorescence in situ hybridization (FISH) analysis revealed Her-2 amplification (5/126, $4 \%$ ), and immunohistochemistry showed complete PTEN loss $(4 / 155,3 \%)$ and estrogen receptor or progesterone receptor expression anomalies (114/242, 47\%).

\section{Disease response to phase I trial therapy}

Two hundred forty-two patients received 358 types of therapy under 94 different phase I clinical trials conducted at MD Anderson during the period studied. Among these 242 patients, 160 received therapy as a participant in 1 phase I clinical trial, 48 from 2 phase I clinical trials, and 34 from 3 or more clinical trials. The 94 phase I clinical trials were classified as follows: singleagent targeted therapy $(n=33)$, combined targeted therapy $(n=31)$, targeted therapy plus chemotherapy $(n=22)$, chemotherapy alone $(n=7)$, and immunotherapy $(n=1)$. The 358 therapies were grouped as matched therapy ( $\mathrm{n}$ $=59)$ or unmatched/known therapy $(\mathrm{n}=299)$, and antiangiogenic therapy $(\mathrm{n}=224)$ or non-anti-angiogenic therapy $(\mathrm{n}=134)$.

The overall objective response rate across the 358 therapies in our analysis was $9 \%$ (complete response: $\mathrm{n}=4,1 \%$; partial response: $\mathrm{n}=30,8 \%$ ) and the stable disease rate was $45 \%(n=162)$, as shown in Figure 1 . The median duration of response was 8.6 months. No difference in objective response was observed between matched (9\% objective response across 59 therapies) and unmatched/known therapy $(10 \%$ objective response across 299 therapies; $p=0.77)$. Targeted therapy plus chemotherapy was associated with a significantly higher objective response $(20 / 112,18 \%)$ than other types of therapy $(14 / 246,6 \% ; p<0.001)$. The objective response rate observed in trials using a bevacizumab-based regimen $(16 / 89,18 \%)$, had a significantly better response rate than other regimens $(p=0.019)$ : VEGFR-TKI-based regimens (4/54, 7\%), bevacizumab plus VEGFR-TKIs $(2 / 27,7 \%)$, and non-anti-angiogenic therapies (12/188, 6\%). No patients experiencing multiple lines of therapy on phase I trials of anti-angiogenic therapies had symptoms and/or radiographic findings of small bowel obstruction.

\section{PFS}

The median PFS of 358 phase I treats delivered in 242 consecutive patients with recurrent HG-EOC 
Table 1: Characteristics of the 242 patients enrolled in phase I clinical trials during the period studied

\begin{tabular}{|c|c|}
\hline Characteristic & No. $(\%)$ \\
\hline Median age (range) & 64 years $(27-79$ years $)$ \\
\hline \multicolumn{2}{|l|}{ Race/ethnicity } \\
\hline White & $194(80)$ \\
\hline African-American & $20(8)$ \\
\hline Hispanic & $12(5)$ \\
\hline Asian & $16(7)$ \\
\hline \multicolumn{2}{|l|}{ Pathologic diagnosis (all high-grade) } \\
\hline Serous carcinoma & $192(79)$ \\
\hline Clear cell carcinoma & $24(10)$ \\
\hline Poorly differentiated/undifferentiated carcinoma & $19(8)$ \\
\hline Endometrioid carcinoma & $7(3)$ \\
\hline \multicolumn{2}{|l|}{ Initial disease stage } \\
\hline I-II & $23(10)$ \\
\hline III-IV & $219(90)$ \\
\hline \multicolumn{2}{|l|}{ Eastern Cooperative Oncology Group performance status } \\
\hline 0 & $66(27)$ \\
\hline$\geq 1$ & $176(73)$ \\
\hline \multicolumn{2}{|l|}{ Lactate dehydrogenase } \\
\hline Normal & $143(59)$ \\
\hline Above normal & $99(41)$ \\
\hline \multicolumn{2}{|l|}{ Albumin } \\
\hline$\geq 3.5 \mathrm{~g} / \mathrm{dL}$ & $214(88)$ \\
\hline$<3.5 \mathrm{~g} / \mathrm{dL}$ & $28(12)$ \\
\hline \multicolumn{2}{|l|}{ Metastatic sites } \\
\hline$\leq 2$ & $156(64)$ \\
\hline$>2$ & $86(36)$ \\
\hline \multicolumn{2}{|l|}{ Prior anti-angiogenic therapy } \\
\hline Yes & $123(51)$ \\
\hline No & $119(49)$ \\
\hline \multicolumn{2}{|l|}{ Prior systemic therapy } \\
\hline 1 prior line & $21(9)$ \\
\hline 2 prior lines & $30(12)$ \\
\hline$\geq 3$ prior lines & $191(79)$ \\
\hline Median no. of prior therapies (range) & $4(1-16)$ \\
\hline \multicolumn{2}{|l|}{ No. of phase I protocols enrolled } \\
\hline 1 & $161(67)$ \\
\hline$\geq 2$ & $81(33)$ \\
\hline
\end{tabular}


who received their phase I clinical trial therapy at our phase I trial clinic was 3 months (95\% confidence interval [95\% CI] , 2.6-3.4 months). Univariate analyses demonstrated that the prolonged PFS after treatment in a phase I clinical trial was associated with normal albumin levels ( $p=0.005), 2$ or fewer metastatic sites $(p=0.049)$, and prior anti-angiogenic therapy $(p=0.028)$. Patients receiving bevacizumab-based regimens $(n=89$ therapies $)$ had significantly longer PFS (4.2 months) than those receiving other therapies $(p=0.017)$, including VEGFRTKI-based regimens ( $\mathrm{n}=54$ therapies, 2.9 months), bevacizumab plus VEGFR-TKIs $(\mathrm{n}=27$ therapies, 2.6 months), and non-anti-angiogenic therapy ( $\mathrm{n}=188$ therapies, 2.8 months), as shown in Figure 2.

Patients who received targeted therapy combined with chemotherapy had a median PFS of 4.1 months, which compared favorably ( $p=0.074)$ with other strategies, including single-agent targeted therapy (2.4 months), combination of targeted therapies (2.9 months), and chemotherapy alone (2.5 months). Preliminary data revealed that phase I clinical trial therapy with bevacizumab and nabpaclitaxel plus gemcitabine, Aurora A kinase inhibitor, or Aurora A kinase inhibitor plus paclitaxel led to a median PFS of greater than 6 months.

The multivariable Cox proportional hazards model, which included the factors of age ( $<60$ years or $\geq 60$ years), race/ethnicity (white or nonwhite), pathologic diagnosis (serous carcinoma or other), ECOG performance status (0 or $\geq 1$ ), LDH (normal or above normal), albumin $(\geq 3.5$ $\mathrm{g} / \mathrm{dL}$ [normal] or $<3.5 \mathrm{~g} / \mathrm{dL}$ ), number of metastatic sites $(\leq 2$ or $>2$ ), prior anti-angiogenic therapy (yes or no), previous lines of systemic therapy $(<3$ or $\geq 3$ ), first-time delivery of therapy in a phase I clinical trial (yes or no), and therapy match of the treatment in the phase I clinical trial (matched or unmatched/known), showed that age $<60$ years $(p=0.022)$, albumin $\geq 3.5 \mathrm{~g} / \mathrm{dL}(p=0.007)$, and no prior anti-angiogenic therapy $(p=0.007)$ were independent predictors of prolonged PFS.

OS

In all 242 patients who received therapy in a phase I clinical trial, a median OS of 38.2 months from the initial clinic visit observed, which was similar to that of patients referred to our clinic who did not receive treatment in a phase I clinical trial $(\mathrm{n}=83,40.9$ months; $p=0.12)$. Among the patients who received therapy, a median OS of 44.3 months from the date of initial recurrence was observed in patients who received subsequent therapies with bevacizumab and VEGFR-TKI-based therapies, which compared favorably, although not statistically significant ( $p=0.64)$, with those who had only 1 line of anti-angiogenic therapy (36.4 months) or no antiangiogenic therapy (32.7 months).

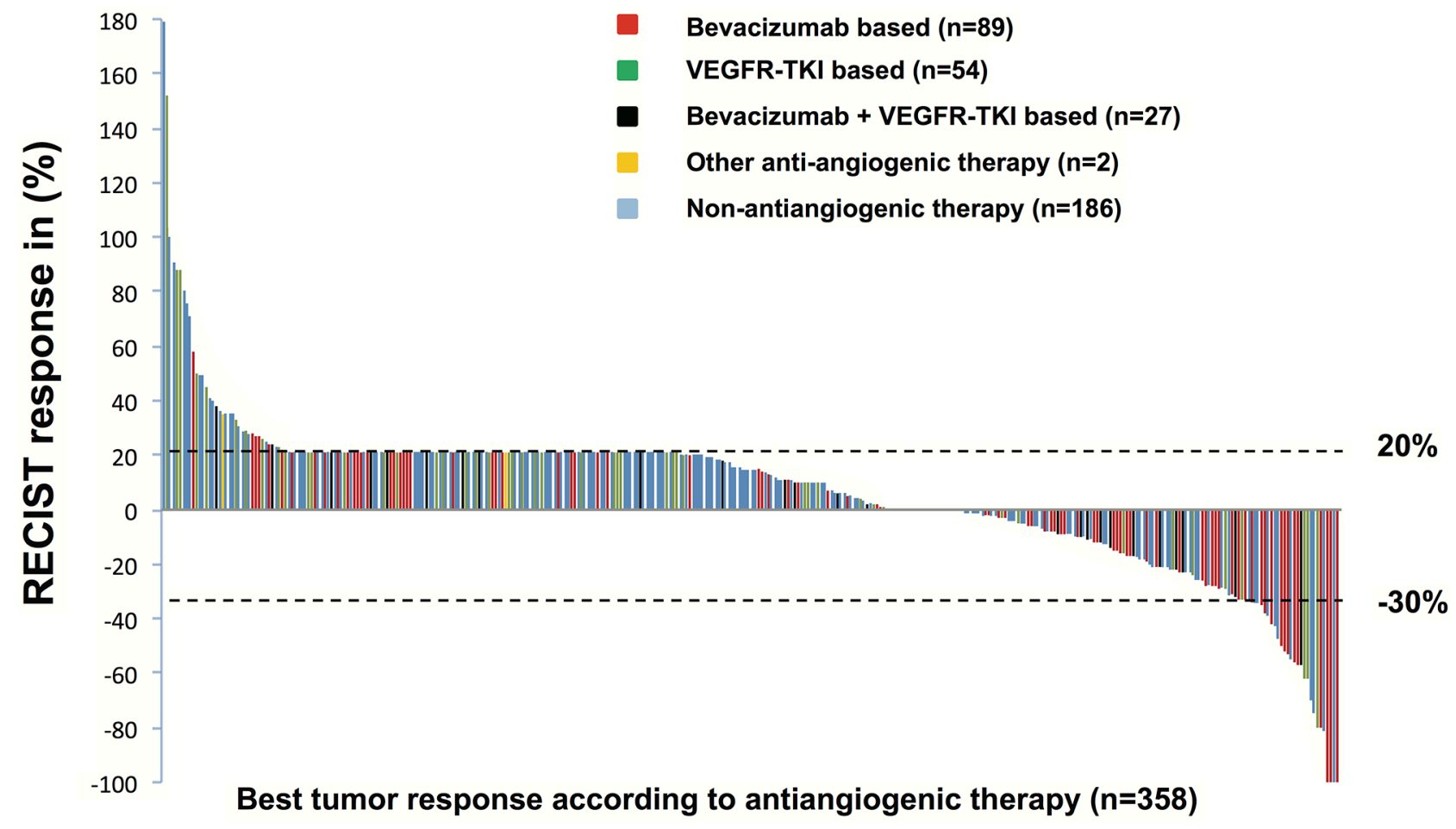

Figure 1: Waterfall plot shows the best objective responses according to Response Evaluation Criteria in Solid Tumors (RECIST). All 358 phase I clinical trial therapies administered to 242 patients are shown. A $21 \%$ or higher RECIST response represents new lesions, early tumor progression, or early withdrawal from treatment for other reasons; this may be arbitrarily designated $21 \%$ or higher disease progression or actual tumor progression of $21 \%$ or higher. VEGFR-TKI, vascular endothelial growth factor receptor-tyrosine kinase inhibitor. 
Univariate analyses of factors affecting OS from initiation of the first therapy in a phase I clinical trial $(\mathrm{n}=$ 242 patients) revealed that normal LDH levels ( $p=0.009)$, albumin $\geq 3.5 \mathrm{~g} / \mathrm{dL}(p<0.001), \leq 2$ metastatic sites $(p<$ $0.001)$, and no prior anti-angiogenic therapy $(p=0.013)$ were associated with prolonged OS (Table 2). Patients receiving therapy in 3 or more phase I clinical trials had a median OS of 23.8 months, which was significantly better $(p<0.001)$ than those receiving therapy in 2 trials (10.4 months) or only 1 trial (7.7 months), as shown in Figure 3A. Patients who received VEGFR-TKI therapy $(\mathrm{n}=79)$ had a median OS of 12.2 months, which was significantly longer than the median OS of 8.6 months in those who receive non-VEGFR-TKI therapy ( $\mathrm{n}=163, p=$ 0.015 ), as shown in Figure $3 \mathrm{~B}$.

The multivariable Cox proportional hazards model, which included the factors of age ( $<60$ years or $\geq 60$ years), race/ethnicity (white or nonwhite), pathologic diagnosis (serous carcinoma or other), ECOG performance status (0 or $\geq 1$ ), LDH (normal or above normal), albumin ( $\geq 3.5 \mathrm{~g}$ /
$\mathrm{dL}$ or $<3.5 \mathrm{~g} / \mathrm{dL}$ ), number of metastatic sites ( $\leq 2$ or $>2$ ), prior anti-angiogenic therapy (yes or no), and previous lines of systemic therapy ( $<3$ or $\geq 3$ ) showed that age $<60$ years $(p=0.029)$, normal LDH levels $(p=0.047)$, albumin $\geq 3.5 \mathrm{~g} / \mathrm{dL}(p<0.001), \leq 2$ metastatic sites $(p<0.001)$, and no prior anti-angiogenic therapy $(\mathrm{p}=0.015)$ were independent factors predicting prolonged OS (Table 3 ).

\section{DISCUSSION}

In the current study, we assessed a large cohort of patients with recurrent HG-EOC who were referred to the phase I clinical trials program at MD Anderson. Approximately $74 \%$ of the patients referred to the program during the period studied participated in a therapeutic phase I clinical trial. We found that anti-angiogenic therapy was one of the most important strategies for the treatment of HG-EOC. Chemotherapy plus bevacizumabbased or Aurora A kinase inhibitor-based regimens were potentially effective and yielded a median PFS of more

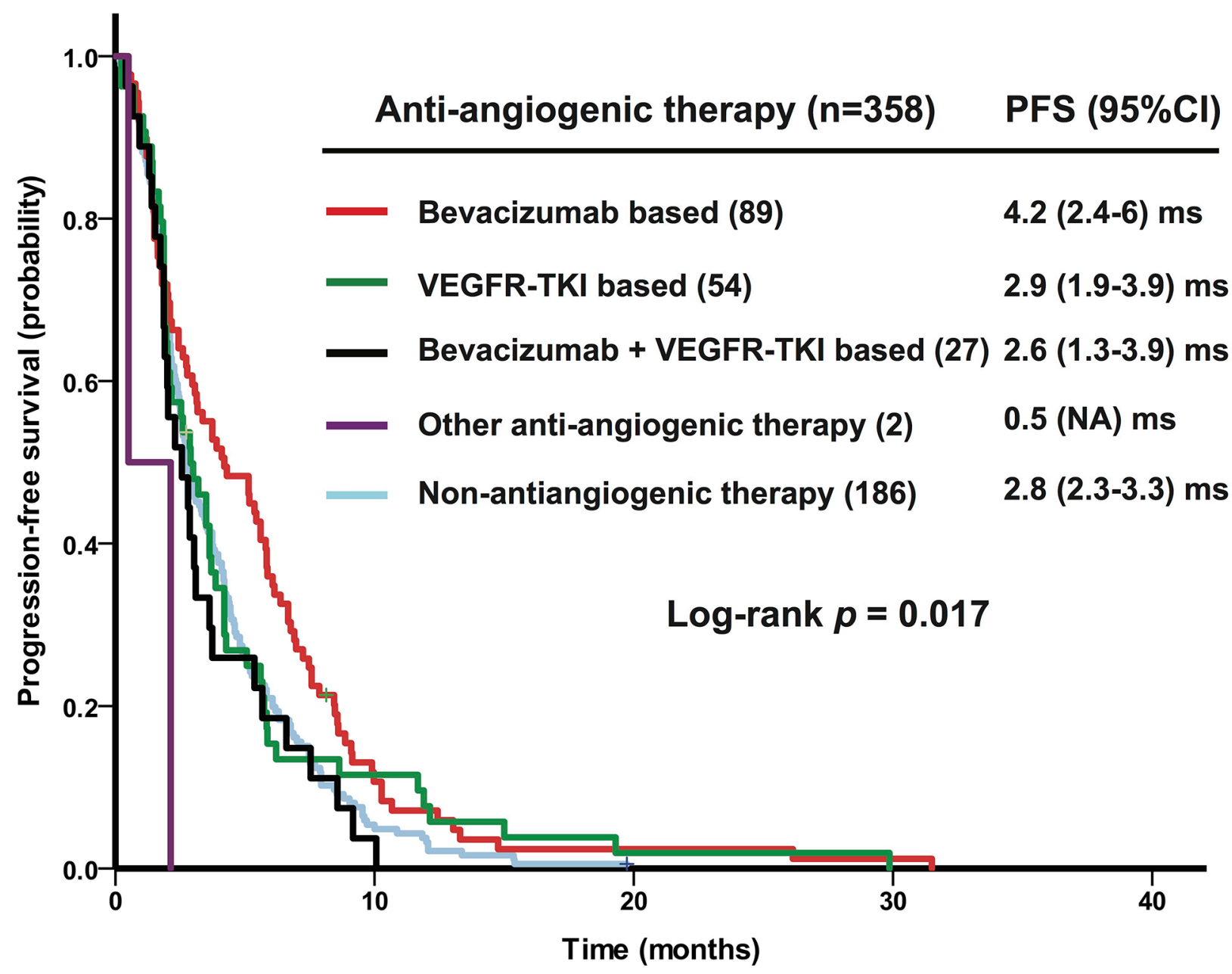

Figure 2: Kaplan-Meier plot shows progression-free survival (PFS) after phase I therapies (358 phase I clinical trial therapies) administered to 242 patients with recurrent high-grade epithelial ovarian cancer according to the type of therapy. VEGFR-TKI, vascular endothelial growth factor receptor-tyrosine kinase inhibitor. 
Table 2: Univariate analyses of overall survival (OS) after treatment in a phase I clinical trial $(n=242$ patients)

\begin{tabular}{|c|c|c|}
\hline Factor & $\begin{array}{c}\text { OS }(95 \% \text { confidence interval }) \text {, } \\
\text { months }\end{array}$ & $p$ \\
\hline Age & & 0.536 \\
\hline$<60$ years & $9.4(8.5-10.3)$ & \\
\hline$\geq 60$ years & $9.3(7.0-11.6)$ & \\
\hline Race/ethnicity & & 0.947 \\
\hline White & $9.4(8.2-10.6)$ & \\
\hline Nonwhite & $8.7(6.8-10.6)$ & \\
\hline Pathologic diagnosis & & 0.265 \\
\hline Serous carcinoma & $9.4(8.3-10.6)$ & \\
\hline Other & $9.2(7.1-11.3)$ & \\
\hline Eastern Cooperative Oncology Group performance status & & 0.165 \\
\hline 0 & $10.1(8.3-11.8)$ & \\
\hline$\geq 1$ & $8.7(7.7-9.7)$ & \\
\hline Lactate dehydrogenase & & 0.009 \\
\hline Normal & $11.1(9.9-12.4)$ & \\
\hline Above normal & $8.1(7.0-9.2)$ & \\
\hline Albumin & & $<0.001$ \\
\hline$\geq 3.5 \mathrm{~g} / \mathrm{dL}$ (normal) & $10.1(9.0-11.2)$ & \\
\hline$<3.5 \mathrm{~g} / \mathrm{dL}$ & $3.9(3.0-4.7)$ & \\
\hline Metastatic sites & & $<0.001$ \\
\hline$\leq 2$ & $10.6(8.9-12.3)$ & \\
\hline$>2$ & $7.6(5.2-9.9)$ & \\
\hline Prior anti-angiogenic therapy & & 0.013 \\
\hline Yes & $8.6(7.1-10.1)$ & \\
\hline No & $11.0(8.8-13.2)$ & \\
\hline Prior systemic therapy & & 0.075 \\
\hline$<3$ lines & $12.0(8.4-15.7)$ & \\
\hline$\geq 3$ lines & $8.7(7.8-9.6)$ & \\
\hline
\end{tabular}

than 6 months. Our preliminary evidence indicates that continuous anti-angiogenic therapy after tumor progression is associated with a significant survival benefit, and eligible patients should continue to participate in phase I clinical trials even if anti-angiogenic therapy from the first trial is not effective.

The tumor suppressor gene TP53 is mutated in approximately $96 \%$ of cases of high-grade serous ovarian carcinoma [33]. TP53 is at the hub of numerous signaling pathways involved in tumorigenesis, tumor development, and metastasis $[34,35]$, and it is triggered by a range of cellular stresses, such as transient cell cycle arrest, DNA repair, senescence, apoptosis, metabolism, stem cell maintenance, invasion, metastasis, and communication with the tumor microenvironment [36-38]. Mutations in TP53 in cancer cells lead to accelerated tumor growth as a result of increased VEGF expression and neovascularization [39]. These processes represent an important survival pathway [40, 41], and VEGF inhibition in patients with p53-mutant malignancies is therefore an effective therapeutic strategy $[12,13]$. Bevacizumab, a monoclonal anti-VEGF-A antibody, has been shown to improve PFS when combined with chemotherapy compared with chemotherapy alone in several phase III studies, such as the ICON7 [9, 11], OCEANS [10], AURELIA [8], GOG218 [12], and GOG213 [5] trials. Likewise, in our study, anti-angiogenic therapies showed remarkable antitumor activity in the phase I clinical trials 

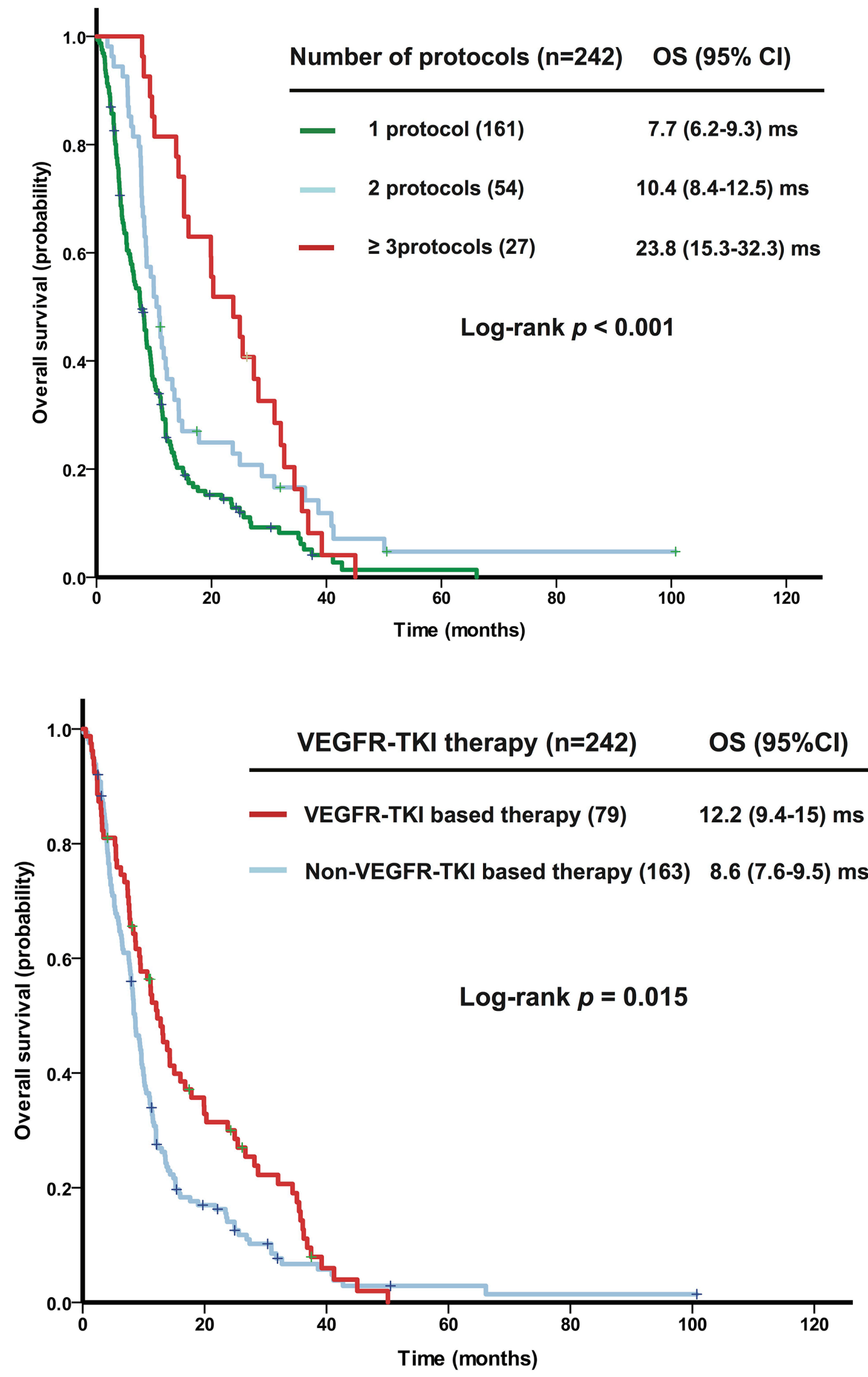

Figure 3: Kaplan-Meier plots shows overall survival (OS) in patients with recurrent high-grade epithelial ovarian cancer $(\mathrm{n}=$ 242) according to A. the number of therapies received in phase I clinical trials and B. the type of therapy received (vascular endothelial growth factor receptor-tyrosine kinase inhibitor [VEGFR-TKI]-based therapy or other). 
Table 3: Multivariate analyses of overall survival after treatment in a phase I clinical trial $(n=242$ patients)

\begin{tabular}{lcccc}
\hline Factor & $\boldsymbol{p}$ & Hazard ratio & \multicolumn{2}{c}{$\mathbf{9 5 \%}$ confidence interval } \\
\cline { 4 - 5 } & & & Lower & Upper \\
\hline Age $<60$ years & 0.029 & 1.374 & 1.034 & 1.827 \\
White & 0.511 & 1.126 & 0.790 & 1.606 \\
Serous carcinoma & 0.109 & 1.324 & 0.939 & 1.866 \\
ECOG performance status $\geq 1$ & 0.310 & 1.174 & 0.861 & 1.599 \\
Normal lactate dehydrogenase levels & 0.047 & 1.342 & 1.004 & 1.795 \\
Albumin $\geq 3.5$ g/dL (normal) & $<0.001$ & 3.060 & 1.987 & 4.712 \\
$\leq 2$ metastatic sites & $<0.001$ & 1.637 & 1.229 & 2.179 \\
Prior antiangiogenic therapy & 0.015 & 0.704 & 0.530 & 0.935 \\
$<3$ prior lines of systemic therapy & 0.644 & 1.091 & 0.755 & 1.576 \\
\hline
\end{tabular}

and were associated with increased PFS, demonstrating the importance of $\mathrm{p} 53$-VEGF crosstalk in ovarian cancer tumorigenesis and development and the therapeutic advantage of VEGF inhibition in this cohort of patients.

In the current study, we sought to determine whether prior exposure to bevacizumab-based therapy potentially affected the antitumor activity of further treatment with bevacizumab-based chemotherapeutic regimens in phase I studies. No difference was observed in overall objective responses and PFS between patients with prior bevacizumab exposure who received bevacizumab-based chemotherapeutic regimens in phase I clinical studies and those without prior exposure. Furthermore, continuation of anti-angiogenic therapy in combination with various other therapeutic agents after the patient had experienced tumor progression led to a median OS of 12.2 months, which was significantly greater than the median OS in those who did not continue anti-angiogenic therapy (8.6 months). These results are consistent with those of previous studies showing that maintenance of antiangiogenic therapy with sequential VEGF inhibition-based regimens after disease progression has a clinical benefit in patients with advanced malignancies [42, 43]. This could be explained by the following reasons, as well as others: 1) the pro-angiogenic property of p53-VEGF crosstalk is essential to ovarian cancer tumorigenesis, development, and resistance to cancer therapy; 2) bevacizumab targets a common pathway mediating resistance to multiple cancer therapeutic agents, and therefore continuous treatment with bevacizumab sensitizes the cancer cells to subsequent therapeutic regimens; 3) because VEGF suppresses the tumor immune microenvironment, prolonged treatment with bevacizumab improves tumor response, leading to better antitumor activity in subsequent therapies; or 4) no or little overlapping toxicity between bevacizumab and other cancer therapeutic agents allows treatment with bevacizumab to be continued in subsequent lines of therapy. Moreover, we found that the more phase I clinical trials that patients participated in, the longer their overall survival duration. This finding suggests that patients with metastatic or recurrent HG-EOC should be encouraged to participate in more phase I clinical trials whenever they are eligible. Another explanation might be that the patients who lived longer got more treatment.

Our current data revealed no difference in antitumor activity between matched and unmatched therapy. This finding may simply indicate that the presence of TP53 or other mutations predominantly in HG-EOC dilutes the therapeutic advantage of matched therapy, or that the genomic alterations targeted by the matched therapy reported here are not genuine driver mutations. In addition to anti-angiogenic therapy, another therapeutic strategy for HG-EOC is to target the Aurora kinase family, which plays a critical role in the regulation of chromosomal segregation and cytokinesis during mitotic progression. Aurora A kinase overexpression has been strongly linked with poor clinical outcomes, providing a potential therapeutic target in HG-EOC. In our study, treatment with an Aurora A kinase inhibitor as a single agent or in combination with chemotherapy produced promising antitumor activity, warranting further exploration.

In considering the clinical relevance and importance of our findings, several limitations and nuances should be noted. First, as is always inherent to retrospective methodology, the selection bias of patient referral to our phase I clinical trials program may limit the generalizability of our findings. The rate of phase I clinical trial enrollment for patients with HG-EOC (74\%) is significantly higher than the average patient enrollment rate in phase I trials at our institution $(\sim 55 \%)$, as we previously described [44]. Second, small sample sizes in the subgroup analyses limit the validity of these statistical assessments. Finally, the actual reasons for declining to enroll in a phase I trial were unknown or not identified in the electronic medical records of patients in the period studied. Therefore, conclusions from this retrospective study should be considered preliminary evidence for the purpose of hypothesis generation, and these findings 
require further validation in larger prospective studies to confirm the benefit of continuously use of anti-angiogenic therapy after tumor progression.

In summary, we retrospectively reviewed the records of 242 consecutive patients with HG-EOC who received therapy in a phase I clinical trial. To the best of our knowledge, this research represents the largest retrospective study in patients with metastatic or recurrent HG-EOC who were referred to a phase I clinical trial. Our study revealed the potential efficacy and importance of therapeutic strategies to target angiogenesis and Aurora A kinase, as single agents or in combination with chemotherapy, for the treatment of HG-EOC. Preliminary data indicated that continuous VEGF inhibition in combination with various other therapeutic agents was associated with improved clinical outcomes. Patients with advanced HG-EOC who are eligible should be encouraged to try novel therapeutic regimens in clinical trials.

\section{MATERIALS AND METHODS}

\section{Patients}

Three hundred twenty-five consecutive patients with histologically confirmed HG-EOC, including high-grade papillary serous, poorly differentiated, endometrioid, and clear cell carcinoma, were referred to the phase I clinical trials program at MD Anderson between May 1, 2006, and December 31, 2014. Among these patients, we reviewed the records of the 242 consecutive patients (74\%) who had actually received treatment as a participant in at least 1 phase I clinical trial during the period studied. We obtained patient demographics, medical history, Eastern Cooperative Oncology Group (ECOG) performance status, laboratory results, gene aberration status, and status or outcome of treatment administered in the phase I clinical trial. Trial procedures, data collection, and the subsequent analysis were performed in accordance with the guidelines of the MD Anderson Institutional Review Board.

\section{Molecular analysis}

Molecular profiling via next-generation sequencing has only recently become available. When adequate tissue samples were available for patients in the period studied, molecular analyses were performed. DNA was extracted from microdissected paraffin-embedded tumor specimens and gene aberrations were detected at the Clinical Laboratory Improvement Amendments-certified MD Anderson Molecular Diagnostics Laboratory [28, 29].

\section{Treatment and evaluation}

The decision to enroll an eligible patient into a phase I clinical trial varied over time, depending on the protocol availability and the preference of the treating physician. The treatment in a phase I clinical trial was considered matched therapy if at least 1 drug in the regimen was known to inhibit the functional activity of at least 1 of the patient's gene aberrations. Toxic effects were assessed according to the National Cancer Institute Common Terminology Criteria for Adverse Events version 3.0 or 4.0 [30], and tumor response was evaluated according to the Response Evaluation Criteria in Solid Tumors version 1.0 or 1.1 [31, 32], depending on the individual protocol. The usual surveillance for tumor treatment effect was performed every other cycle (one every 6 or 8 weeks). Progression-free survival (PFS) was calculated from the date of initiation of treatment in a phase I clinical trial to the first objective documentation of disease progression, the date of death, or the last date censored as of September 30, 2015. Overall survival (OS) was calculated from the date of the date of initiation of treatment in a phase I clinical trial to the date of death or the last date censored as of September 30, 2015.

\section{Statistical analysis}

Patient characteristics, including age, race/ethnicity, pathologic type, disease stage, ECOG performance status, lactate dehydrogenase (LDH) levels, albumin levels, number of metastatic sites, previous systemic treatment, prior anti-angiogenic therapy, therapy match of the treatment in the phase I clinical trial, number of phase I clinical trials in which the patient enrolled, and gene aberrations were summarized using frequency distributions and percentages. A waterfall plot analysis was used to illustrate antitumor efficacy according to the type of treatment delivered in the phase I clinical trial (anti-angiogenic or non-anti-angiogenic). Categorical variables were compared via chi-square and Fisher exact tests. Survival durations (PFS and OS) were assessed using Kaplan-Meier curves by the log-rank test. A multivariable Cox proportional hazards model was used for multivariate analysis. All tests were 2-sided and considered significant when the $p$ value was less than 0.05 . Statistical analyses were performed using SPSS version 23.0 software (SPSS, Chicago, IL).

\section{ACKNOWLEDGMENTS}

The authors thank patients, faculty, and staff in the Departments of Investigational Cancer Therapeutics and Gynecologic Oncology at The University of Texas MD Anderson Cancer Center for their participation in phase I clinical trials and patient care, and Erica Goodoff in the Department of Scientific Publications at MD Anderson for editing the manuscript. RLC is supported in part by the Ann Rife Cox Chair in Gynecology and the Judy Reis Ovarian Cancer Research fund. This work was 
supported by National Natural Sciences Foundation, China (81101778 and 81472206).

\section{CONFLICTS OF INTEREST}

All authors have no potential conflicts of interest to disclose.

\section{REFERENCES}

1. Siegel RL, Miller KD and Jemal A. Cancer statistics, 2015. CA Cancer J Clin. 2015; 65:5-29.

2. Seidman JD, Horkayne-Szakaly I, Haiba M, Boice CR, Kurman RJ and Ronnett BM. The histologic type and stage distribution of ovarian carcinomas of surface epithelial origin. International journal of gynecological pathology. $2004 ; 23: 41-44$

3. Li J, Fadare O, Xiang L, Kong B and Zheng W. Ovarian serous carcinoma: recent concepts on its origin and carcinogenesis. Journal of hematology \& oncology. 2012; $5: 8$

4. Vergote I, Trope CG, Amant F, Kristensen GB, Ehlen T, Johnson N, Verheijen RH, van der Burg ME, Lacave AJ, Panici PB, Kenter GG, Casado A, Mendiola C, Coens C, Verleye L, Stuart GC, et al. Neoadjuvant chemotherapy or primary surgery in stage IIIC or IV ovarian cancer. N Engl J Med. 2010; 363:943-953.

5. Coleman RL, Monk BJ, Sood AK and Herzog TJ. Latest research and treatment of advanced-stage epithelial ovarian cancer. Nature reviews Clinical oncology. 2013; 10:211-224.

6. Jayson GC, Kohn EC, Kitchener HC and Ledermann JA. Ovarian cancer. Lancet. 2014; 384:1376-1388.

7. Diaz-Montes TP and Bristow RE. Secondary cytoreduction for patients with recurrent ovarian cancer. Current oncology reports. 2005; 7:451-458.

8. Pujade-Lauraine E, Hilpert F, Weber B, Reuss A, Poveda A, Kristensen G, Sorio R, Vergote I, Witteveen P, Bamias A, Pereira D, Wimberger P, Oaknin A, Mirza MR, Follana P, Bollag D, et al. Bevacizumab combined with chemotherapy for platinum-resistant recurrent ovarian cancer: The AURELIA open-label randomized phase III trial. Journal of clinical oncology. 2014; 32:1302-1308.

9. Oza AM, Cook AD, Pfisterer J, Embleton A, Ledermann JA, Pujade-Lauraine E, Kristensen G, Carey MS, Beale P, Cervantes A, Park-Simon TW, Rustin G, Joly F, Mirza MR, Plante M, Quinn M, et al. Standard chemotherapy with or without bevacizumab for women with newly diagnosed ovarian cancer (ICON7): overall survival results of a phase 3 randomised trial. Lancet Oncol. 2015; 16:928-936.

10. Aghajanian C, Blank SV, Goff BA, Judson PL, Teneriello MG, Husain A, Sovak MA, Yi J and Nycum LR. OCEANS: a randomized, double-blind, placebo-controlled phase
III trial of chemotherapy with or without bevacizumab in patients with platinum-sensitive recurrent epithelial ovarian, primary peritoneal, or fallopian tube cancer. Journal of clinical oncology. 2012; 30:2039-2045.

11. Perren TJ, Swart AM, Pfisterer J, Ledermann JA, PujadeLauraine E, Kristensen G, Carey MS, Beale P, Cervantes A, Kurzeder C, du Bois A, Sehouli J, Kimmig R, Stahle A, Collinson F, Essapen S, et al. A phase 3 trial of bevacizumab in ovarian cancer. N Engl J Med. 2011; $365: 2484-2496$.

12. Burger RA, Brady MF, Bookman MA, Fleming GF, Monk BJ, Huang H, Mannel RS, Homesley HD, Fowler J, Greer BE, Boente M, Birrer MJ, Liang SX and Gynecologic Oncology G. Incorporation of bevacizumab in the primary treatment of ovarian cancer. N Engl J Med. 2011; 365:2473-2483.

13. Fu S, Hou MM, Naing A, Janku F, Hess K, Zinner R, Subbiah V, Hong D, Wheler J, Piha-Paul S, Tsimberidou A, Karp D, Araujo D, Kee B, Hwu P, Wolff R, et al. Phase I study of pazopanib and vorinostat: a therapeutic approach for inhibiting mutant p53-mediated angiogenesis and facilitating mutant p53 degradation. Annals of oncology. 2015; 26:1012-1018.

14. Pignata S, Lorusso D, Scambia G, Sambataro D, Tamberi S, Cinieri S, Mosconi AM, Orditura M, Brandes AA, Arcangeli V, Panici PB, Pisano C, Cecere SC, Di Napoli M, Raspagliesi F, Maltese G, et al. Pazopanib plus weekly paclitaxel versus weekly paclitaxel alone for platinumresistant or platinum-refractory advanced ovarian cancer (MITO 11): a randomised, open-label, phase 2 trial. The Lancet Oncology. 2015; 16:561-568.

15. du Bois A, Floquet A, Kim JW, Rau J, del Campo JM, Friedlander M, Pignata S, Fujiwara K, Vergote I, Colombo N, Mirza MR, Monk BJ, Kimmig R, Ray-Coquard I, Zang $\mathrm{R}$, Diaz-Padilla I, et al. Incorporation of pazopanib in maintenance therapy of ovarian cancer. Journal of clinical oncology. 2014; 32:3374-3382.

16. Friedlander M, Hancock KC, Rischin D, Messing MJ, Stringer CA, Matthys GM, Ma B, Hodge JP and Lager JJ. A Phase II, open-label study evaluating pazopanib in patients with recurrent ovarian cancer. Gynecologic oncology. 2010; 119:32-37.

17. Ledermann J, Harter P, Gourley C, Friedlander M, Vergote I, Rustin G, Scott C, Meier W, Shapira-Frommer R, Safra T, Matei D, Macpherson E, Watkins C, Carmichael J and Matulonis U. Olaparib maintenance therapy in platinumsensitive relapsed ovarian cancer. The New England journal of medicine. 2012; 366:1382-1392.

18. Ledermann J, Harter P, Gourley C, Friedlander M, Vergote I, Rustin G, Scott CL, Meier W, Shapira-Frommer R, Safra T, Matei D, Fielding A, Spencer S, Dougherty B, Orr M, Hodgson D, et al. Olaparib maintenance therapy in patients with platinum-sensitive relapsed serous ovarian cancer: a preplanned retrospective analysis of outcomes by BRCA 
status in a randomised phase 2 trial. The Lancet Oncology. 2014; 15:852-861.

19. Liu JF, Barry WT, Birrer M, Lee JM, Buckanovich RJ, Fleming GF, Rimel B, Buss MK, Nattam S, Hurteau J, Luo W, Quy P, Whalen C, Obermayer L, Lee H, Winer EP, et al. Combination cediranib and olaparib versus olaparib alone for women with recurrent platinum-sensitive ovarian cancer: a randomised phase 2 study. The Lancet Oncology. 2014; 15:1207-1214.

20. Fu S, Hennessy BT, Ng CS, Ju Z, Coombes KR, Wolf JK, Sood AK, Levenback CF, Coleman RL, Kavanagh JJ, Gershenson DM, Markman M, Dice K, Howard A, Li J, Li $\mathrm{Y}$, et al. Perifosine plus docetaxel in patients with platinum and taxane resistant or refractory high-grade epithelial ovarian cancer. Gynecologic oncology. 2012; 126:47-53.

21. Moroney J, Fu S, Moulder S, Falchook G, Helgason T, Levenback C, Hong D, Naing A, Wheler J and Kurzrock R. Phase I study of the antiangiogenic antibody bevacizumab and the mTOR/hypoxia-inducible factor inhibitor temsirolimus combined with liposomal doxorubicin: tolerance and biological activity. Clinical cancer research. 2012; 18:5796-5805.

22. Farley J, Brady WE, Vathipadiekal V, Lankes HA, Coleman R, Morgan MA, Mannel R, Yamada SD, Mutch D, Rodgers WH, Birrer M and Gershenson DM. Selumetinib in women with recurrent low-grade serous carcinoma of the ovary or peritoneum: an open-label, single-arm, phase 2 study. The Lancet Oncology. 2013; 14:134-140.

23. McNeish IA, Ledermann JA, Webber L, James L, Kaye SB, Hall M, Hall G, Clamp A, Earl H, Banerjee S, Kristeleit R, Raja F, Feeney A, Lawrence C, Dawson-Athey L, Persic $\mathrm{M}$, et al. A randomised, placebo-controlled trial of weekly paclitaxel and saracatinib (AZD0530) in platinum-resistant ovarian, fallopian tube or primary peritoneal cancerdagger. Annals of oncology. 2014; 25:1988-1995.

24. Matsuo K, Nishimura M, Bottsford-Miller JN, Huang J, Komurov K, Armaiz-Pena GN, Shahzad MM, Stone RL, Roh JW, Sanguino AM, Lu C, Im DD, Rosenshien NB, Sakakibara A, Nagano T, Yamasaki M, et al. Targeting SRC in mucinous ovarian carcinoma. Clinical cancer research. 2011; 17:5367-5378.

25. Do K, Wilsker D, Ji J, Zlott J, Freshwater T, Kinders RJ, Collins J, Chen AP, Doroshow JH and Kummar S. Phase I Study of Single-Agent AZD1775 (MK-1775), a Wee1 Kinase Inhibitor, in Patients With Refractory Solid Tumors. Journal of clinical oncology. 2015; 33:3409-3415.

26. Venkatakrishnan K, Kim TM, Lin CC, Thye LS, Chng WJ, Ma B, Chen MH, Zhou X, Liu H, Kelly V and Kim WS. Phase 1 study of the investigational Aurora A kinase inhibitor alisertib (MLN8237) in East Asian cancer patients: pharmacokinetics and recommended phase 2 dose. Investigational new drugs. 2015; 33:942-953.

27. Matulonis UA, Sharma S, Ghamande S, Gordon MS, Del Prete SA, Ray-Coquard I, Kutarska E, Liu H, Fingert H,
Zhou X, Danaee H and Schilder RJ. Phase II study of MLN8237 (alisertib), an investigational Aurora A kinase inhibitor, in patients with platinum-resistant or -refractory epithelial ovarian, fallopian tube, or primary peritoneal carcinoma. Gynecologic oncology. 2012; 127:63-69.

28. Hou MM, Liu X, Wheler J, Naing A, Hong D, Coleman RL, Tsimberidou A, Janku F, Zinner R, Lu K, Kurzrock $\mathrm{R}$ and $\mathrm{Fu} \mathrm{S}$. Targeted PI3K/AKT/mTOR therapy for metastatic carcinomas of the cervix: A phase I clinical experience. Oncotarget. 2014; 5:11168-11179. doi: 10.18632/ oncotarget.2584.

29. Hou MM, Liu X, Wheler J, Naing A, Hong D, Bodurka D, Schmeler K, Tsimberidou A, Janku F, Zinner R, PihaPaul S, Hu CY, Lu K, Kurzrock R and Fu S. Outcomes of patients with metastatic cervical cancer in a phase I clinical trials program. Anticancer research. 2014; 34:2349-2355.

30. Cancer Therapy Evaluation Program NCI;Common Terminology Criteria for Adverse events (CTCAE), accessed on 16 December 2015: http://ctep.cancer.gov/ protocolDevelopment/electronic_applications/ctc.htm.

31. Therasse P, Arbuck SG, Eisenhauer EA, Wanders J, Kaplan RS, Rubinstein L, Verweij J, Van Glabbeke M, van Oosterom AT, Christian MC and Gwyther SG. New guidelines to evaluate the response to treatment in solid tumors. European Organization for Research and Treatment of Cancer, National Cancer Institute of the United States, National Cancer Institute of Canada. J Natl Cancer Inst. 2000; 92:205-216.

32. Eisenhauer EA, Therasse P, Bogaerts J, Schwartz LH, Sargent D, Ford R, Dancey J, Arbuck S, Gwyther S, Mooney M, Rubinstein L, Shankar L, Dodd L, Kaplan R, Lacombe D and Verweij J. New response evaluation criteria in solid tumours: revised RECIST guideline (version 1.1). European journal of cancer. 2009; 45:228-247.

33. Cancer Genome Atlas Research N. Integrated genomic analyses of ovarian carcinoma. Nature. 2011; 474:609-615.

34. Muller PA and Vousden KH. Mutant p53 in cancer: new functions and therapeutic opportunities. Cancer cell. 2014; 25:304-317.

35. Leroy B, Fournier JL, Ishioka C, Monti P, Inga A, Fronza $\mathrm{G}$ and Soussi T. The TP53 website: an integrative resource centre for the TP53 mutation database and TP53 mutant analysis. Nucleic acids research. 2013; 41(Database issue):D962-969.

36. Yamakuchi M, Lotterman CD, Bao C, Hruban RH, Karim B, Mendell JT, Huso D and Lowenstein CJ. P53-induced microRNA-107 inhibits HIF-1 and tumor angiogenesis. Proceedings of the National Academy of Sciences of the United States of America. 2010; 107:6334-6339.

37. Vogelstein B and Kinzler KW. Cancer genes and the pathways they control. Nat Med. 2004; 10:789-799.

38. Bieging KT, Mello SS and Attardi LD. Unravelling mechanisms of p53-mediated tumour suppression. Nat Rev Cancer. 2014; 14:359-370. 
39. Ambs S, Merriam WG, Ogunfusika MO, Bennett WP, Ishibe N, Hussain SP, Tzeng EE, Geller DA, Billiar TR and Harris CC. p53 and vascular endothelial growth factor regulate tumor growth of NOS2-expressing human carcinoma cells. Nat Med. 1998; 4:1371-1376.

40. Joshi H, Bhanot G, Borresen-Dale AL and Kristensen V. Potential tumorigenic programs associated with TP53 mutation status reveal role of VEGF pathway. British journal of cancer. 2012; 107:1722-1728.

41. Montero E, Abreu C and Tonino P. Relationship between VEGF and p53 expression and tumor cell proliferation in human gastrointestinal carcinomas. Journal of cancer research and clinical oncology. 2008; 134:193-201.

42. Bennouna J, Sastre J, Arnold D, Osterlund P, Greil R, Van Cutsem E, von Moos R, Vieitez JM, Bouche O, Borg C,
Steffens CC, Alonso-Orduna V, Schlichting C, ReyesRivera I, Bendahmane B, Andre T, et al. Continuation of bevacizumab after first progression in metastatic colorectal cancer (ML18147): a randomised phase 3 trial. Lancet Oncol. 2013; 14:29-37.

43. Grothey A, Sugrue MM, Purdie DM, Dong W, Sargent D, Hedrick E and Kozloff M. Bevacizumab beyond first progression is associated with prolonged overall survival in metastatic colorectal cancer: results from a large observational cohort study (BRiTE). Journal of clinical oncology. 2008; 26:5326-5334.

44. Fu S, McQuinn L, Naing A, Wheler JJ, Janku F, Falchook GS, Piha-Paul SA, Tu D, Howard A, Tsimberidou A, Zinner R, Hong DS and Kurzrock R. Barriers to study enrollment in patients with advanced cancer referred to a phase I clinical trials unit. The oncologist. 2013; 18:1315-1320. 